\title{
Produção e qualidade de propágulos de morangueiro em diferentes concentrações de nitrogênio no cultivo sem solo
}

\author{
Clarisse Silva Oliveira ${ }^{1}$, Carine Cocco², Jerônimo Luiz Andriolo*3 ${ }^{3}$, Dilson Antônio Bisognin ${ }^{3}$, \\ Lígia Erpen ${ }^{4}$, Gustavo Giménez Franquez ${ }^{5}$
}

\section{RESUMO}

O objetivo deste trabalho foi avaliar o efeito da concentração de nitrogênio na produção e na qualidade de mudas de raízes nuas e pontas de estolões de morangueiro no cultivo sem solo. O plantio das matrizes foi realizado em 13 de setembro de 2007, e a colheita das mudas em 18 de fevereiro de 2008. Os tratamentos foram quatro concentrações de $\mathrm{N}$ na solução nutritiva de $8,11,14$ e $17 \mathrm{mmol} \mathrm{L}^{-1}$. O delineamento utilizado foi o inteiramente casualizado, com cinco repetições de 3,6 $\mathrm{m}^{2}$. No momento da colheita foram determinados o número e o diâmetro da coroa das mudas de raízes nuas e o número de pontas de estolões. As diferentes concentrações de $\mathrm{N}$ não afetaram o número de mudas e de pontas de estolões, cujas médias foram de 339 e 836, respectivamente. O diâmetro da coroa das mudas de raízes nuas diminuiu linearmente com o aumento da concentração de $\mathrm{N}$ na solução nutritiva. Concluiu-se que o aumento da concentração de $\mathrm{N}$ na solução nutritiva, no sistema de cultivo sem solo com substrato de areia, não afeta o número de mudas de raízes nuas e nem de pontas de estolões, mas reduz o diâmetro da coroa das mudas de raízes nuas de morangueiro.

Palavra-chave: Fragaria x ananassa Duch, hidroponia, mudas, substrato.

\section{ABSTRACT}

\section{Production and quality of strawberry transplants in different nitrogen concentrations in soilless growing system}

The objective of this work was to evaluate the effects of nitrogen concentration on the production and quality of strawberry bare root transplants and runner tips in soilless growing system. Stock plants were planted on September $13^{\text {th }}$, 2007. Transplants were harvested on February $18^{\text {th }}$, 2008. Nitrogen concentrations of 8,11, 14 and $17 \mathrm{mmol} \mathrm{L}^{-1}$ in the nutrient solution were evaluated. The experiment was arranged in a complete randomized design with five replications of $3.6 \mathrm{~m}^{2}$ plots. Number and crown diameter of bare root transplants and number of runner tips were evaluated at harvest. Number of bare root transplants and runner tips was not affected by $\mathrm{N}$ concentration in the nutrient solution. An average of 339 bare root transplants and 836 runner tips were harvest per stock plant. Increasing $1 \mathrm{mmol}$ of $\mathrm{N}$ concentration of the nutrient solution decreases the crown diameter of bare root transplants in $0.4 \mathrm{~mm}$. In the soilless grown system with sand as substrate, the $\mathrm{N}$ concentration does not affect the production of bare root transplants and runner tips, but do affect crown diameter of strawberry bare root transplants.

Key words: Fragaria x ananassa Duch., hydroponics, transplants substrate.

Recebido para publicação em outubro de 2008 e aprovado em dezembro de 2009

${ }^{1}$ Engenheira-Agrônoma, Mestre. Universidade Federal de Santa Maria (UFSM), Departamento de Fitotecnia, Centro de Ciências Rurais (CCR), Programa de Pós-graduação em Agronomia, Av. Roraima, 1000, Campus Universitário. 97105-900 Santa Maria, RS, Brasil. clarisoliveira@yahoo.com.br

2 Engenheira-Agrônoma, Mestranda. Universidade Federal de Santa Maria (UFSM), Departamento de Fitotecnia, Centro de Ciências Rurais (CCR), Programa de Pós-graduação em Agronomia, Av. Roraima, 1000, Campus Universitário. 97105-900 Santa Maria, RS, Brasil. carinecocco@yahoo.com.br

${ }^{3}$ Engenheiros-Agrônomos. Ph. Doctor. UFSM, Departamento de Fitotecnia, CCR. Av. Roraima, 1000, Campus Universitário. 97105-900 Santa Maria, RS, Brasil. andriolo@smail.ufs.br, dilsonb@smail.ufsm.br. *Autor correspondente

${ }^{4}$ Graduanda de Engenharia Agronômica. Universidade Federal de Santa Maria (UFSM), Centro de Ciências Rurais (CCR), Av. Roraima, 1000, Campus Universitário. 97105-900 Santa Maria, RS, Brasil. ligia_erpen@yahoo.com.br

${ }^{5}$ Engenheiro-Agrônomo, Doutor. INIA Las Brujas. E.E. Wilson Ferreira Aldunate. Ruta 48. Km 10. C.C. 33085. Canelones. Uruguai. ggimenez@lb.inia.org.uy 


\section{INTRODUÇÃO}

Um dos aspectos atualmente limitantes à produtividade do morangueiro no Brasil é a escassez de mudas de elevadas qualidades fisiológica e sanitária. A fase de produção de mudas é crucial dentro da cadeia produtiva do morangueiro, pela necessidade de renovação anual das lavouras de produção de frutas. Os gastos com a aquisição de mudas podem representar até $45 \%$ do custo de produção .

No método tradicional de produção de mudas de morangueiro o plantio das matrizes é feito no solo. A taxa de propagação é baixa, as mudas obtidas são desuniformes e a contaminação por doenças é elevada, principalmente pela antracnose (Colletotrichum spp.) (Santos \& Medeiros, 2003). O emprego de sistemas de cultivo sem solo é a alternativa indicada para atingir altas produtividade, qualidade e sanidade das mudas dessa cultura (Calvete et al. 2002; Giménez, 2008). Esse sistema pode ser empregado tanto para a produção de mudas de raízes nuas quanto de pontas de estolões, para posterior produção de mudas com torrão em bandejas (Lieten, 1998; Durner et al. 2002; Giménez, 2008). Um sistema de cultivo sem solo empregando substrato mineral ou orgânico e com reduzido consumo de energia elétrica foi desenvolvido para a produção de mudas e de frutas do morangueiro (Andriolo, 2007).

Diversas formulações de soluções nutritivas podem ser encontradas na literatura para o cultivo sem solo do morangueiro, tanto na fase de produção de mudas quanto de frutas (Giménez et al., 2008). No Brasil, são escassos os resultados de pesquisas sobre a composição e o manejo da solução nutritiva na produção de mudas dessa cultura.

Dentre os nutrientes minerais, o nitrogênio é o que mais afeta o crescimento e desenvolvimento da planta do morangueiro. Na fase de estolonamento, a deficiência de $\mathrm{N}$ afeta tanto o comprimento quanto o número de ramificações dos estolões (Deng \& Woodward, 2002). Resultados disponíveis na literatura indicam que níveis elevados de nitrogênio favorecem a emissão precoce e aumentam o número de estolões e de ramificações da coroa e diminuem o comprimento dos estolões (Tworkoski et al., 2001). O aumento no número de ramificações da coroa é favorecido por níveis moderados de $\mathrm{N}$ logo após o plantio (Hennion \& Veschambre, 1997).

O diâmetro da coroa é a principal variável que tem sido empregada para avaliar a qualidade das mudas de morangueiro, a qual afeta tanto a precocidade como a produtividade de frutas. Resultados da literatura indicam que as mudas de raízes nuas devem ter um diâmetro de coroa de pelo menos $8 \mathrm{~mm}$ para obter elevada produtividade de frutas na lavoura (Hochmuth et al., 2006). Com relação às pontas de estolões, informações desse tipo não foram encontradas na literatura.
O objetivo deste trabalho foi avaliar o efeito da concentração de nitrogênio na solução nutritiva na produção de mudas de raízes nuas e de pontas de estolões e também na qualidade das mudas de raízes nuas.

\section{MATERIAL E MÉTODOS}

O experimento foi realizado entre setembro de 2007 e fevereiro de 2008, no interior de um abrigo de $150 \mathrm{~m}^{2}$, coberto com polietileno de baixa densidade de $150 \mu \mathrm{m}$ de espessura, no Departamento de Fitotecnia da Universidade Federal de Santa Maria.

As unidades experimentais foram formadas por telhas de fibrocimento com 3,6 m de comprimento, 1,10 m de largura, canais de 0,06 m de altura e 0,18 m de distância entre os canais. Cada telha foi apoiada sobre suportes a $0,80 \mathrm{~m}$ de altura do solo, com declividade de $1 \%$, e a superfície foi revestida com filme transparente de polietileno de baixa densidade, com espessura de $100 \mu \mathrm{m}$. Os canais foram preenchidos com brita basáltica média, de tamanho de partículas entre 0,015 e 0,020 m. Sobre a brita foi colocada uma tela anti-inseto e sobre a tela uma camada de $0,15 \mathrm{~m}$ de areia média, como substrato para o crescimento das raízes das plantas. As características físicas da areia foram determinadas no Laboratório de Física do Solo da UFSM, indicando granulometria entre 0,001 e 0,003 m, densidade aparente de $1,7 \mathrm{~g} \mathrm{~cm}^{-3}$ e capacidade de retenção de água de 198,6 mL dm³ ${ }^{3}$. Um reservatório de fibra de vidro com capacidade para $500 \mathrm{~L}$ foi instalado próximo à extremidade inferior das telhas, para armazenar a solução nutritiva (Andriolo, 2007).

A solução nutritiva de referência foi aquela descrita em Hennion \& Veschambre (1997), com a composição em mmol L-1 de 11,0 de $\mathrm{NO}_{3}^{--} ; 2$ de $\mathrm{H}_{2} \mathrm{PO}_{4}^{-} ; 1$ de $\mathrm{SO}_{4}^{-2} ; 6,15$ de $\mathrm{K}^{+} ; 3$ de $\mathrm{Ca}^{+2}$; 1 de $\mathrm{Mg}^{2+}$; 0 ,43 de $\mathrm{NH}_{4}^{+}$. Os micronutrientes foram fornecidos nas concentrações de, em $\mathrm{mg} \mathrm{L}^{-1}: 0,03$ de Mo; 0,42 de B; 0,06 de Cu; 0,50 de Mn; 0,22 de Zn; e 1,0 de Fe. Os tratamentos foram constituídos por quatro concentrações de N na solução nutritiva: 8 (T1), 11 (T2), 14 (T3) e 17 (T4) mmol L-1 , sendo T2 a solução de referência (Hennion \& Veschambre, 1997). O delineamento experimental empregado foi inteiramente casualizado, com cinco repetições, sendo cada parcela composta por uma unidade do dispositivo de cultura. As concentrações dos demais nutrientes não foram alteradas. Para atingir a composição desejada em cada tratamento, foram empregados nitrato de potássio $\left(\mathrm{KNO}_{3}\right)$, nitrato de amônio $\left(\mathrm{NH}_{4} \mathrm{NO}_{3}\right)$, nitrato de cálcio $\left[\left(\mathrm{CaNO}_{3}\right)_{2}\right]$ (calcinit), monofosfato de potássio $\left(\mathrm{KH}_{2} \mathrm{PO}_{4}\right)$, sulfato de potássio $\left(\mathrm{K}_{2} \mathrm{SO}_{4}\right)$ e sulfato de magnésio $\left(\mathrm{MgSO}_{4}\right)$, conforme o caso. O cálculo das relações iônicas entre os macronutrientes e das quantidades de sais foi feito de acordo com a metodologia descrita por Andriolo (1999). 
A CE e o $\mathrm{pH}$ foram medidos diariamente, e os valores mantidos entre 1,3 e 1,7 dS m ${ }^{-1}$ e 5,5 a 6,5, respectivamente, em todos os tratamentos. A CE foi corrigida mediante adição de água ou de volumes complementares de solução nutritiva e o pH, mediante adição de soluções $1 \mathrm{~N}$ de $\mathrm{H}_{3} \mathrm{PO}_{4}$ ou $\mathrm{KOH}$, com volumes estimados a partir de uma curva de titulação previamente ajustada no laboratório. Volumes complementares de solução nutritiva preparada de acordo com a composição original de cada tratamento foram adicionados sempre que o volume medido no reservatório foi igual ou inferior a 50\% do volume original. Para tal, uma relação linear foi previamente ajustada entre a altura da coluna líquida e o volume contido no reservatório. Nenhum descarte de solução nutritiva foi feito durante o período experimental.

A frequência das fertirrigações foi estimada com base na capacidade de retenção de água do substrato e a transpiração potencial da cultura, de forma a fornecer diariamente volumes de água superiores àqueles transpirados, com coeficiente de drenagem igual ou superior a $30 \%$. A transpiração potencial foi estimada considerando-se a radiação solar global incidente no topo da cobertura vegetal e a área foliar da cultura (Hennion \& Veschambre, 1997), com base em dados de literatura sobre determinações similares feitas em hortaliças cultivadas nos mesmos local e ambiente (Dalmago et al., 2006). Por meio dessa estimativa, foram programadas quatro fertirrigações diárias de $15 \mathrm{~min}$, às $9,11,13$ e $16 \mathrm{~h} 30 \mathrm{~min}$, controladas por um programador horário.

As temperaturas máximas do ar no interior do telado foram medidas diariamente por meio de um termômetro de máximas instalado no interior de um abrigo meteorológico localizado na área central do telado, a 1,5 m de altura. No ambiente externo, foram medidas na Estação Climatológica do mesmo Departamento, localizado a aproximadamente 50 m da área experimental.

Foram utilizadas mudas matrizes do clone SM-INIA LBD 15.1, oriundas de micropropagação realizada no Laboratório de Melhoramento e Propagação Vegetativa de Plantas do mesmo Departamento. A aclimatização foi feita em bandejas com areia no mesmo local onde foi conduzido o experimento. O plantio das mudas matrizes foi realizado em 13 de setembro de 2007, na densidade de uma planta $\mathrm{m}^{-2}$. O experimento foi encerrado em 18 de fevereiro de 2008. O controle de pragas e doenças foi feito de acordo com as indicações técnicas de Antunes \& Duarte Filho (2005). Durante a condução do experimento, as pontas de estolões que ultrapassaram os limites laterais de cada unidade experimental foram retiradas e contadas.

Ao final do experimento, as mudas de raízes nuas originadas de pontas de estolões que enraizaram no substrato foram arrancadas, contadas e o diâmetro da coroa medido com paquímetro. As pontas de estolões com diâmetro de coroa superior a $2 \mathrm{~mm}$ foram extraídas e contadas. A esses resultados foram somadas as quantidades retiradas anteriormente em cada unidade experimental. As plantas-matrizes originais foram colhidas separadamente e o crescimento foi determinado pela massa seca total da planta após secagem em estufa de circulação forçada de ar, na temperatura de $65{ }^{\circ} \mathrm{C}$, até obter massa constante entre duas determinações consecutivas. Os resultados foram submetidos à análise de variância, e as variáveis com diferenças significativas pelo teste $\mathrm{F}$ a $5 \%$ de probabilidade foram submetidas à análise de regressão polinomial.

\section{RESULTADOS E DISCUSSÃO}

Os valores semanais de condutividade elétrica nas diferentes concentrações de $\mathrm{N}$ apresentaram variações ao longo do período experimental (Figura 1). As médias foram de 1,5; 1,5; 1,6; e 1,7 dS m ${ }^{1}$, em T1, T2, T3 e T4, respectivamente. Essas flutuações foram observadas tanto no decorrer do período quanto entre os tratamentos. No decorrer do período podem ser atribuídas principalmente à dinâmica de absorção dos nutrientes, em consequência do crescimento e do acúmulo de massa de matéria seca pela cultura. Entre as diferentes doses de $\mathrm{N}$, as flutuações foram pequenas e situaram-se dentro do limite previsto no protocolo experimental. Os valores médios durante todo o período do experimento situaram-se dentro da faixa entre 1,4 e 1,8 dS m ${ }^{-1}$, considerada a mais favorável ao crescimento e à produtividade do morangueiro (Lieten, 1998; Sarooshi \& Cresswell, 1994; Paranjpe et al., 2003).

Não houve diferença no número de mudas de raízes nuas e de pontas de estolões com diâmetro superior a 2 mm produzidas nas diferentes concentrações de $\mathrm{N}$ da solução nutritiva. A média foi de 339 mudas de raízes nuas e 836 pontas de estolões para cada planta matriz. Considerando que cada ponta de estolão tem o potencial para originar uma muda com torrão, isso representa uma taxa de propagação de 1.175 mudas por planta matriz. Nos Esta-

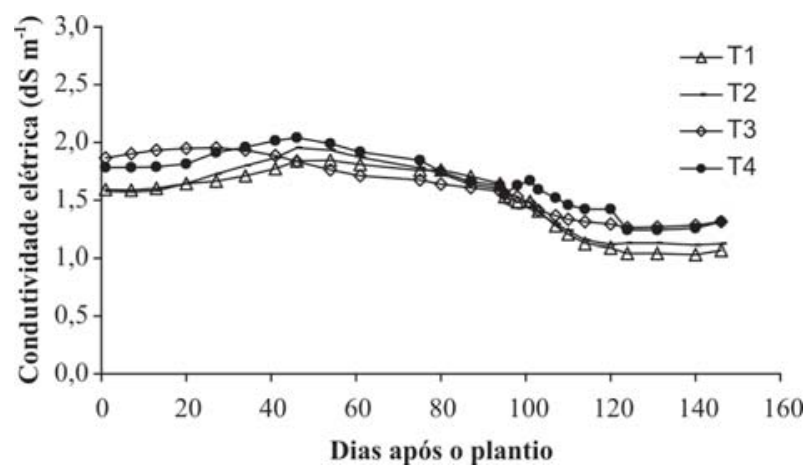

Figura 1. Médias semanais da condutividade elétrica da solução nutritiva no decorrer do experimento de produção de mudas de morangueiro com concentrações de nitrogênio de 8, 11, 14 e 17 mmol L-1. 
dos Unidos, Hokanson et al. (2004) e Takeda et al. (2004) obtiveram cerca de 500 pontas de estolões por planta matriz. Em Pelotas (RS), Oliveira et al. (2007) empregaram recipientes suspensos em casa de vegetação e relataram a produção de 34 mudas de raízes nuas por planta matriz do cultivar Dover e de 31,9 do cultivar Campinas e 27,4 pontas de estolões considerando ambos os cultivares. Em Piracicaba (SP), também em ambiente protegido e com recipientes suspensos, Tessarioli Neto (2001) obteve a produção de 55 mudas de raízes nuas por planta matriz do cultivar Dover e 44 do cultivar Campinas. Em Santa Maria, nas mesmas condições de produção do atual experimento Giménez (2008) obteve anteriormente 145 mudas de raízes nuas e 590 pontas de estolões do clone SM-INIA LBD 15.1 em bancadas de areia fertirrigadas com solução nutritiva.

As diferentes taxas de propagação do morangueiro podem ser atribuídas às diferenças genéticas entre clones e cultivares, às diferenças ambientais e de sistemas de cultivo e ao próprio manejo da cultura. A produção de estolão é um caráter genético e, portanto, característico de cada clone ou cultivar (Tworkoski et al., 2001; Serçe $\&$ Hancock, 2005). A fase de estolonamento é controlada principalmente pela temperatura. Consequentemente, tanto a amplitude térmica diária quanto a duração do período térmico favorável a esse processo afetam o número de estolões produzidos. As características do sistema de cultivo, especialmente quanto à disponibilidade de nutrientes minerais, podem interferir tanto no crescimento das plantas matrizes quanto no estolonamento (Tworkoski et al., 2001).

As concentrações de $\mathrm{N}$ na solução nutritiva influenciaram o crescimento das plantas matrizes (Figura 2). As médias ajustaram-se a um modelo polinomial com máxima acumulação de massa de matéria seca estimada na concentração de $\mathrm{N}$ de 12,8 mmol L-1. O diâmetro da coroa das mudas de raízes nuas também foi afetado pela concentração de N (Figura 3). O efeito foi linear com coeficiente

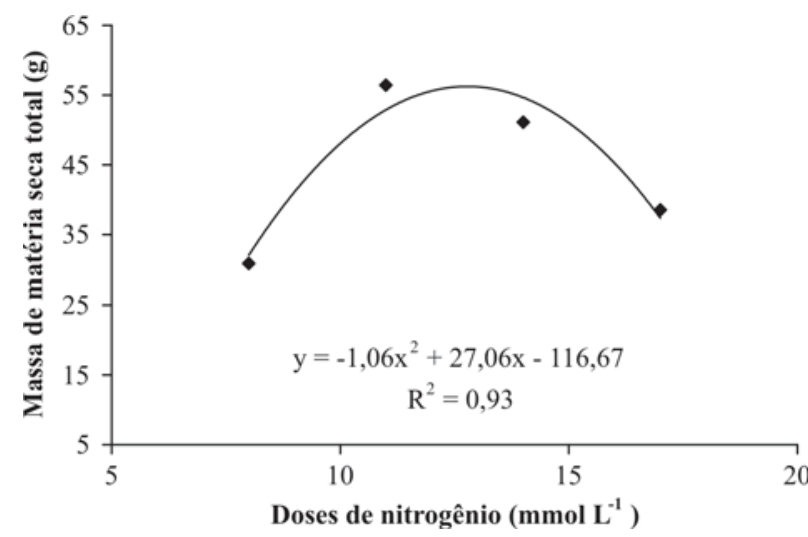

Figura 2. Massa de matéria seca total da planta matriz de mudas de morangueiro em sistema de cultivo sem solo com concentrações de nitrogênio de 8, 11, 14 e $17 \mathrm{mmol} \mathrm{L}^{-1}$ na solução nutritiva. angular negativo. A redução estimada no diâmetro da coroa por efeito do $\mathrm{N}$ foi de $0,4 \mathrm{~mm}$ para cada unidade de acréscimo da concentração. O maior diâmetro foi de 9 mm, atingido nas mudas fertirrigadas com a solução contendo $8 \mathrm{mmol} \mathrm{L}^{-1}$ de N. O menor foi de 7,8 mm, ocorrido na concentração de $17 \mathrm{mmol}^{-1}$. Apesar da diminuição do diâmetro da coroa com o aumento da concentração de N, o valor de 7,8 mm está próximo do considerado mínimo para uma muda de boa qualidade fisiológica, que é de $8 \mathrm{~mm}$ (Hochmuth et al., 2006).

O acúmulo de soma térmica é uma das variáveis que pode explicar a elevada taxa de propagação obtida nesse experimento. As temperaturas do ar foram mais elevadas no interior do ambiente telado, com médias das máximas superiores em $5^{\circ} \mathrm{C}$ àquelas do ambiente externo. As temperaturas mais elevadas foram favoráveis ao estolonamento, que ocorre a partir de temperaturas entre 22 e $26^{\circ} \mathrm{C}$ (Santos \& Medeiros, 2003). Essa hipótese encontra sustentação na precocidade do início do estolonamento, o qual foi constatado aos 13 dias após o plantio. A precocidade implica em um maior período de tempo para emissão e crescimento de estolões, conduzindo a uma maior taxa de propagação ao final desse período.

O crescimento das mudas avaliado pelo diâmetro da coroa (Figura 3) apresentou tendência negativa e distinta $(p<0,5)$ daquela relativa ao crescimento da planta matriz (Figura 2). Na literatura, o crescimento do compartimento reprodutivo da planta tem sido considerado como dependente do compartimento vegetativo (Heuvelink \& Körner, 2001). Decorre dessa concepção que quanto maior for a produção de fotoassimilados nas folhas mais elevado será o potencial de produtividade, pois a força de dreno das estruturas reprodutivas é mais elevada do que a das vegetativas (González-Real et al., 2008). Na planta do morangueiro a frutificação é substituída pelo estolonamento à medida que aumenta a temperatura do ar. Ambas as fases caracterizam um processo reprodutivo. Os resultados sugerem que ao entrar na última fase a planta do morangueiro altera a relação fonte-dreno entre esses dois compartimentos. O estolão teria crescimento autotrófico logo nas fases iniciais de desenvolvimento após a emissão, com pequena ou nenhuma dependência dos assimilados provenientes da planta matriz. Essa hipótese deve ser testada em novas pesquisas, pois pode determinar os procedimentos a serem adotados na produção de mudas com torrão.

A diminuição observada no crescimento das plantas matrizes sem efeito significativo no número de mudas e uma diminuição linear no diâmetro da coroa das mudas de raízes nuas contraria os resultados de Tworkoski et al. (2001). Em outras culturas, disponibilidades elevadas de $\mathrm{N}$ induziram maior crescimento vegetativo e retardamento da tuberização em batata (Andriolo, 2006; Cogo, 2006) e retardamento da frutificação em plantas de melão (Fogaça, 2007). 


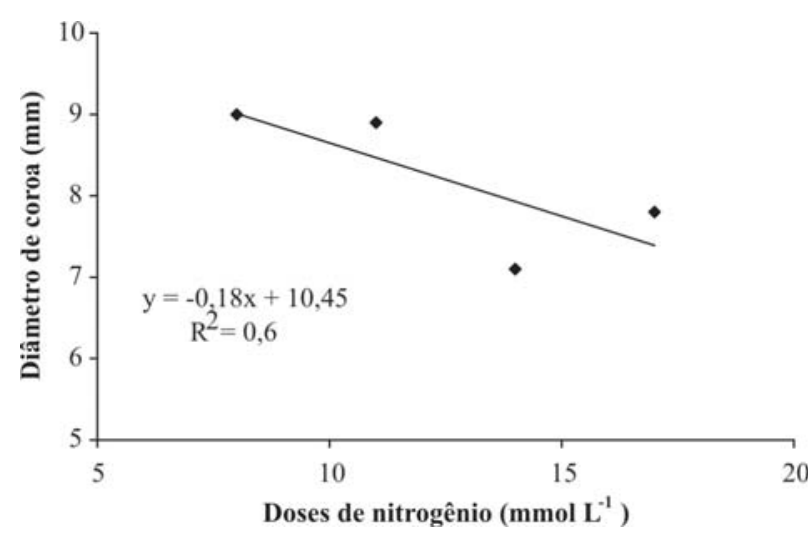

Figura 3. Diâmetro da coroa das mudas de morangueiro em sistema de cultivo sem solo com concentrações de nitrogênio de 8, 11, 14 e 17 mmol L-1 na solução nutritiva.

Essas diferenças podem ser atribuídas ao hábito de crescimento da planta do morangueiro na fase de propagação vegetativa. À medida que prossegue a emissão e o crescimento dos estolões aumenta a densidade de plantas na superfície em torno da planta matriz. A área foliar dessas novas plantas originadas dos estolões cresce até atingir a saturação do IAF e da intercepção da radiação solar. Nessa fase, cada planta considerada individualmente entra em equilíbrio isométrico e uma competição se estabelece entre o crescimento das folhas e a emissão de novos estolões, os quais, quando jovens, têm o crescimento dependente dos assimilados da planta matriz. Consequentemente, os estolões jovens podem morrer por falta de assimilados, e aqueles na fase autotrófica podem ter o crescimento reduzido pela baixa disponibilidade de radiação solar, que pode ser interceptada. Nas doses de N mais elevadas, com intenso crescimento vegetativo, essa competição ocorreria mais precocemente, reduzindo o crescimento posterior tanto da planta matriz quanto dos estolões.

A diminuição do diâmetro da coroa das mudas de raízes nuas com o aumento da disponibilidade de $\mathrm{N}$ (Figura 3) também pode ser consequência do maior e mais precoce crescimento vegetativo das plantas. Para fins de produção de mudas dessa cultura em sistema sem solo, os resultados sugerem que o manejo da disponibilidade de $\mathrm{N}$ deve ser feito levando-se em conta variáveis como o cultivar, época de plantio, arranjo e/ou densidade de plantas. Neste experimento foram empregadas bancadas na superfície das quais enraizaram as plantas de raiz nua originadas dos primeiros estolões. Sistemas individualizados em sacolas onde as plantas fossem repicadas em outro local poderiam diminuir ou evitar o crescimento excessivo da área foliar e a consequente competição entre as plantas. Nessa situação, é possível que um efeito sobre a produção de mudas fosse observado em consequência do diferimento da disponibilidade de $\mathrm{N}$ na solução nutritiva.

\section{CONCLUSÃO}

No sistema de cultivo sem solo com uso de areia como substrato, o aumento da concentração de $\mathrm{N}$ na solução nutritiva não afetou o número de mudas de raízes nuas e nem de pontas de estolão, mas sim o diâmetro da coroa das mudas de raízes nuas de morangueiro.

\section{AGRADECIMENTOS}

Ao Conselho Nacional de Desenvolvimento Científico e Tecnológico - CNPq, pelo auxílio financeiro, processo $n^{\circ} 470177 / 2006-3$, bolsas de pesquisa a Jerônimo Luiz Andriolo e Dílson Antônio Bisognin. À Fundação de Amparo à Pesquisa do RS - FAPERGS, pela Bolsa de Iniciação Científica a Lígia Erpen, processo nº7509443.

\section{REFERÊNCIAS}

Andriolo JL (1999) Fisiologia das culturas protegidas. Santa Maria, UFSM, 142p.

Andriolo JL (2006) Sistema hidropônico fechado com subirrigação para produção de minitubérculos de batata. In: Simpósio de melhoramento genético e previsão de epifitias em batata, Santa Maria. Anais, UFSM. 1:26-40.

Andriolo JL (2007) Preparo e manejo da solução nutritiva na produção de mudas e de frutas do morangueiro. In: Seminário sobre o cultivo hidrôponico de morangueiro, Santa Maria. Anais. UFSM, CCR, Departamento de Fitotecnia. 1:41-50.

Antunes LEC\& Duarte Filho J (2005) Sistema de produção do morango. Produção de mudas. Sistemas de produção 5. Pelotas, EMBRAPA CT. Disponível em http://www.cpact.embrapa/sistema/morango. Acessado em 01 de julho de 2010.

Calvete EO; Kampf AN \& Suzin M (2002) Concentração de sacarose no enraizamento in vitro de morangueiro. Horticultura Brasileira, 20:186-191.

CogoCM., Andriolo JL, Bisognin DA, Godoi RS, Bortolotto OC \& Luz GL (2006) Relação potássio-nitrogênio para o diagnóstico e manejo nutricional da cultura da batata. Pesquisa Agropecuária Brasileira, 41:01-06.

Dalmago GA, Heldwein AB, Nied AH, Grimm EL \& Pivetta CR (2006) Evapotranspiração máxima da cultura do pimentão em estufa plástica em função da radiação solar, temperatura, umidade do ar e déficit de saturação do ar. Revista Brasileira de Agrometeorologia, 36:785-792.

Deng X \& Woodward FI (2002) The Growth and Yield Responses of Fragaria ananassa to Elevated $\mathrm{CO}_{2}$ and $\mathrm{N}$ Supply. Annals of Botany, 81:67-71.

Durner EF, Poling EB \& Maas JL (2002) Recent advances in strawberry plug transplant technology. HortTechnology, 12:545-550.

Fogaça MAF, Andriolo JL, Godoi RS, Gieh RFH, Madalóz JCC \& Barros GTA (2007) Concentração de nitrogênio na solução nutritiva na produtividade e qualidade de frutos de melão cultivado em substrato. Ciência Rural, 37:72-78.

Giménez, G. (2008) Seleção e propagação de clones de morangueiro (Fragaria $x$ ananassa Duch.)Tese de Doutorado. Universidade Federal de Santa Maria, Santa Maria, 119p.

Giménez G, Andriolo JL \& Godói RS (2008) Cultivo sem solo no morangueiro. Ciência Rural, 38:273-279.

Hennion B \& Veschambre D (1997) La fraise: maîtrise de la production. Paris, CTIFL. 299p. 
Heuvelink E, \& Körner O (2001) Parthenocarpic fruit growth reduces yield fluctuation and Blossom-end Rot in sweet pepper. Annals of Botany. 88:69-74.

Hokanson SC, Takeda F, Enns JM \& Black BL (2004) Influence of plant storage duration on strawberry runner tip viability and field performance. HortScience, 39:1596-1600.

Hochmuth G, Cantliffe D, Chandler C, Stanley C, Bish E, Waldo E, Legard D \& Duval, J. (2006) Containerized strawberry transplants reduce establishment-period water use and enhance early growth and flowering compared with bare-root plants. HortTechnology, 6:46-54

Lieten F (1998) La fragola in Belgio-Olanda. In: La Fragola verso il 2000. Convegno Nazionale. Verona. Camera di Commercio Industria Artigianato e Agricultura di Verona, 1:83-94.

González-Real MM, Baille A \& Liu HQ (2008) Influence of fruit load on dry matter and N-distribution in sweet pepper plants. Scientia Horticulturae 117:307-315.

Oliveira RP, Brahm RU \& Scivittaro WB. (2007) Produção de mudas de morangueiro em casa-de-vegetação utilizando recipientes suspensos. Horticultura Brasileira 25:107-109.

Paranjpe A, Cantliffei DJ, Lamb EM, Stoffella PJ \& Powell C (2003) Winter strawberry production in greenhouses using soilless substrates: an alternative to methyl bromide soil fumigation. Proceedings of the Florida State for Horticultural Science, 116:98-105.
Santos A M \& Medeiros A R M (eds). (2003) Morango. Produção. Frutas do Brasil, 40. EMBRAPA CT, 81p.

Sarooshi RA \& Cresswell GC (1994) Effects of hydroponic solution composition, electrical conductivity and plant spacing on yield and quality of strawberries. Australian Journal of Experimental Agriculture, 34:529-535.

Serçe S \& Hancock JF (2005) The temperature and photoperiod regulation of flowering and runnering in the strawberries, Fragaria chiloensis, F. virginiana, and F. x ananassa. Scientia Horticulturae, 103:167-177.

Takeda F, Hokanson SC \& Enns JM (2004) Influence of daughter plant weight and position on strawberry transplant production and field performance in annual plasticulture. HortScience, 39:1592-1595.

Tessarioli Neto J (2001) Produção de mudas de morangueiro (Fragaria $x$ ananassa Duch.) sob cultivo protegido. Tese de Livre-Docencia. USP, Escola Superior de Agricultura "Luiz de Queiroz”, Piracicaba, 75p.

Tworkoski TJ, Benassi TE \& Fumiomi Takeda F (2001)The effect of nitrogen on stolon and ramet growth in four genotypes of Fragaria chiloensis L. Scientia Horticulturae, 88:97-106. 